\title{
Crónica de la pandemia en la ciudad de Rosario.
}

\section{Chronicle of the pandemic in the city of Rosario}

\author{
Lucas F. De Candia, Valeria B. Bulla, Ricardo R. Cordone, Laura Saraceni, Andrea V. Montaner, \\ Mariana, Quignard.
}

Carrera de Posgrado de especialización en Medicina General y Familiar. Facultad de Ciencias Médicas. Universidad Nacional de Rosario. Rosario; Argentina.

Autor por correspondencia: Lucas F. De Candia - lucasdecandia@gmail.com Conflicto de intereses: no presenta.

\section{Resumen}

El presente texto se propone revisar las características de las distintas etapas de la pandemia por el nuevo virus SARS-CoV-2 en la ciudad de Rosario hasta el 05 de diciembre de 2020. Las crisis sanitarias configuran situaciones especiales que ponen a prueba el funcionamiento de los sistemas de salud y la capacidad de las y los trabajadores para adaptarse a nuevos y dinámicos escenarios. Asimismo, la actual pandemia exige definiciones claras y contundentes por parte de los gobiernos. La reflexión permanente sobre las respuestas instauradas es fundamental para el diseño de nuevas estrategias en el futuro inmediato.

Palabras clave: COVID-19. Pandemia. Aislamiento social. Crisis sanitaria.

\section{Resumen}

The new SARS-CoV-2 virus gave rise to a pandemic of great impact in terms of morbidity and mortality at an international level. Governments implemented different coping strategies in order to avoid deaths and preserve pre-pandemic normality as much as possible. However, despite all the efforts, the pandemic generated a multidimensional crisis on a global scale. Argentina initially took strong population measures and later on, a response that attempted to respect regional heterogeneities. This text aims to review the characteristics of the pandemic's different stages in the city of Rosario until December 5, 2020. Permanent reflection on the established responses is essential to devise new strategies in the immediate future.

Keywords: COVID-19. Pandemic. Social isolation. Health crisis. 


\section{La pandemia COVID19}

El nuevo virus denominado SARS — CoV—2, reportado el 31/12/19 en China, dio origen a una pandemia de gran impacto a nivel internacional. El reporte inicial surge a partir de casos inusuales de neumonía. En febrero de 2020, la Organización Mundial de la Salud (OMS) define la enfermedad producida por el nuevo virus como COVID19. Se conocen dos antecedentes recientes de epidemias por coronavirus: a) SARS-CoV (2003): también iniciado en China, que generó una epidemia en varios países con aproximadamente 8000 casos y 800 muertes; y b) MERS—CoV (2015): que inició en Arabia Saudita y del que se registraron aproximadamente 2500 casos y 800 muertes, con la ocurrencia ocasional de algunos casos esporádicos.

El espectro clínico de la enfermedad por COVID-19 varía desde formas asintomáticas hasta condiciones clínicas caracterizadas por fallo respiratorio, multi-orgánico y shock séptico requerimiento de atención en unidades de cuidados intensivos (UCI). Una de las primeras publicaciones describía un cuadro clínico caracterizado por fiebre, disnea, tos seca y malestar general. El estudio se realizó con 41 pacientes hospitalizados con confirmación de infección por SARS-Cov—2. Fiebre y tos fueron los síntomas más frecuentes: 98\% y 76\%, respectivamente. Todos los pacientes presentaban en TAC de tórax signos de neumonía.

El 11/3/20, la OMS declaró la pandemia con más de 820 mil casos y más de 40 mil muertes asociadas a infección por SARS-Cov-2.

El trabajo de Qun Li y col., analizando la epidemiología hasta el 22 de enero de 2020 en Wuhan (China), presenta evidencia que confirma la trasmisión humano-humano del nuevo virus, estiman un $\mathrm{R}_{0}$ de $2.2^{\mathrm{A}} \mathrm{y}$ una duplicación de los casos de neumonía asociada al SARS—Cov—2 cada 7.4 días. Finalmente, concluyen que se van a requerir esfuerzos considerables en el resto del mundo para reducir la trasmisión viral y controlar la epidemia.

En la actualidad, se carece de evidencia suficiente en cantidad y calidad para recomendar el uso clínico de tratamientos farmacológicos. En Argentina, el Ministerio de Salud recomienda para el manejo de personas con COVID hospitalización de grupos de riesgo y casos graves, tratamientos de sostén, antibioticoterapia en individuos con sospecha de neumonía y glucocorticoides en casos severos. Esta última indicación se sustenta en la observación en estudios de que la dexametasona utilizada en casos hospitalizados graves con compromiso respiratorio se asocia a reducción de la mortalidad.

Respecto al desarrollo de vacunas contra el SARS-CoV-2, existen numerosos procesos en estado avanzado de estudio de fases clínicas y se proyectan en el mundo posibles inicios de campañas masivas en el corto plazo. Sin embargo, aún falta mucho para imaginar el fin de la pandemia.

Por otro lado, ya se ha descripto un cuadro denominado como "COVID prolongado" ("Long COVID”). El mismo sería una forma que presenta duración mayor de la sintomatología (superior a 4 semanas). Además, existen publicaciones que reportan secuelas post COVID neurológicas, cardiológicas y respiratorias cuyo impacto largo plazo es por ahora desconocido.

Consecuentemente, en muchos países se optó por implementar medidas de distanciamiento social y suspensión de actividades laborales y educativas como estrategia para disminuir el número de nuevos casos y bajar la mortalidad. Dentro de las diferentes medidas no farmacológicas se destacan las sanitarias (rastreo/detección/aislamiento de casos y contactos) y las no sanitarias (cierre escuelas, teletrabajo, suspensión actividades con acumulación de personas, uso masivo de mascarillas, confinamiento, etc). Si bien, dichas medidas dispararon controversias a nivel internacional, la comunidad científica se mantuvo firme en la postura de recomendarlas como intervención efectiva para el afrontamiento de la pandemia.

Por otro lado, la crisis del virus Ébola dejó una enseñanza contundente: los riesgos que supone poner el foco exclusivamente en la respuesta a la epidemia. Un estudio sobre la mortalidad indirecta en Sierra Leona en contexto de epidemia por Ébola concluyó que las muertes por otras causas fueron al menos tan importantes en número como las directamente vinculadas al virus.

A Según los datos del estudio cada paciente con infección por SARS—Cov—2 contagió a otras 2.2 personas.

Número reproductivo básico $\left(\mathrm{R}_{0}\right)$ : es el número de casos secundarios que podría producir un caso en una población susceptible. Esta determinado por la duración del período infeccioso, la probabilidad de infectar a un individuo susceptible durante un contacto y el número de nuevos individuos susceptibles contactados por unidad de tiempo. (Ridenhour, B., Kowalik, J. M., \& Shay, D. K. (2014). Unraveling R0: considerations for public health applications. American journal of public health, 104(2), e32-e41.) 


\section{Las intervenciones del estado Nacional}

El presidente a partir de la confirmación de los primeros casos en Argentina decretó medidas poblacionales:

- EMERGENCIA SANITARIA:B (11/03/20) se amplían facultades del Ministerio de Salud de Nación para intervenir en diferentes áreas, disponer de mayor presupuesto y emitir un Informe diario de actualización epidemiológicas y recomendaciones.

- $\quad$ AislamiENTO SOCIAL, PREVENTIVO Y OBLIGATORIO (ASPO):C (20/03/20) se decreta el confinamiento, sólo están habilitadas actividades esenciales. Esta medida fue aplicada para todo el país. Los objetivos explicitados fueron aplanar la curva de nuevos contagios, concientizar a la población sobre las normas de cuidado y preparar a los servicios de salud con formación del personal y ampliación de la capacidad. En las semanas subsiguientes, la medida fue prorrogándose.

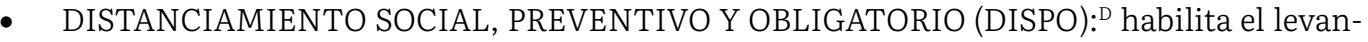
tamiento progresivo de restricciones de actividades en localidades sin circulación comunitaria del virus y con buenos indicadores de tiempo de duplicación de casos y porcentaje de ocupación de camas críticas. La provincia de Santa Fe clasificó para ingresar dentro del DISPO a partir de junio.

\section{Desafíos de la red de salud de Rosario}

La red de salud pública de la ciudad está constituida por efectores dependientes del Ministerio de Salud (MS) de la Provincia de Santa Fe (PSF) y de la Secretaría de Salud Pública de la Municipalidad de Rosario (SSP-MR). La estructura provincial presenta 5 hospitales y 32 centros de salud (CS). La municipal, 6 hospitales, un centro de especialidades médicas ambulatorias, un centro especializado en rehabilitación y 49 CS.

La historia del sector salud en Rosario tuvo un giro de relevancia a partir de la década del ' 90. Los CS de la SSP-MR se constituyeron en instituciones protagónicas en la organización de la red. Algunos de los ejes principales de este proceso fueron la salud como derecho, la Atención Primaria de la Salud (APS) como estrategia y filosofía de la red, la adscripción como política de trabajo, la clínica contextualizante como modelo de atención y el uso de las tecnologías blandas. Así, se buscó poner en el centro de las decisiones a la población, sus necesidades y sus condiciones particulares de vida. Estos valores del proyecto sanitario fueron afianzados y perpetuados en el contexto de la crisis social y económica que sacudió al país en el año 2001. Importantes colectivos de trabajadores de salud protagonizaron este proceso de transformación de la salud pública rosarina. La organización de la respuesta a la actual pandemia en Rosario no debería desconocer su propia historia y los ejes fundadores de la red.

Los reportes publicados sugieren que el nuevo virus SARS-Cov-2 desencadena en el 80\% de los infectados aprox. un cuadro clínico leve-moderado que podría ser diagnosticado y controlado sin ingreso hospitalario. Por otro lado, el restante 20\% necesitan hospitalización y requieren detección precoz y adecuado seguimiento para el acceso oportuno a la internación. En consecuencia, el trabajo de los equipos territoriales debe estar enfocado en detección y aislamiento de casos y contactos, seguimiento ambulatorio aquellos con cuadros leve-moderados e identificación de personas con signos de severidad o pertenecientes a grupos de riesgo.

Sin embargo, el desafío de cuidado integral en la pandemia no se circunscribe a la atención de casos COVID. Como se detalló, la experiencia en otras crisis sanitarias mostró que la tarea del sistema de salud no debe enfocarse sólo en lo relativo a la epidemia. Más allá de los protocolos internacionales y el foco de la prensa mundial puesto en el novedoso virus, múltiples determinantes influyen en los modos de enfermar y morir de las personas en su cotidianeidad. Entonces, la historia particular del

B Decreto de Necesidad y Urgencia 260/2020 (11/3/20) Disponible en: https://www.argentina.gob.ar/coronavirus/dnu

C AISLAMIENTO SOCIAL PREVENTIVO Y OBLIGATORIO Decreto 297/2020 (19/3/20) Disponible en: https://www.boletinoficial.gob.ar/detalleAviso/ primera/227042/20200320. 
sector salud rosarino puede constituirse en una determinación firme en el armado de la estrategia coyuntural. Los equipos de CS conocen las microáreas de mayor vulnerabilidad, cuentan con herramientas para identificar a las personas de riesgo y poseen un vínculo que facilita el cuidado integral y contextualizado.

Resulta innegable la necesidad de ampliar la capacidad instalada de los hospitales. Sin embargo, igual de impostergable es la discusión sobre los modos de acompañar el aislamiento en los territorios más críticos y priorizar la atención de las situaciones más complejas y de mayor riesgo. El ASPO implicó para los equipos territoriales la necesidad de transformar sus procesos de trabajo en pos de un cuidado integral evitando la acumulación de personas. En el plazo de pocas semanas, la mayor parte de los equipos de CS de Rosario se organizaron en función de dos ejes: acompañamiento integral de salud de las personas adscriptas en el marco del ASPO y atención de casos de COVID19. Retomando palabras de Mario Rovere, la apropiación de la APS por parte de los trabajadores de salud es un hecho a destacar y potenciar.

\section{Etapas de la pandemia en Rosario}

Se podrían identificar seis etapas de la pandemia en Rosario que implican diferentes modos de sociabilidad y desafíos para la organización de los equipos de salud.

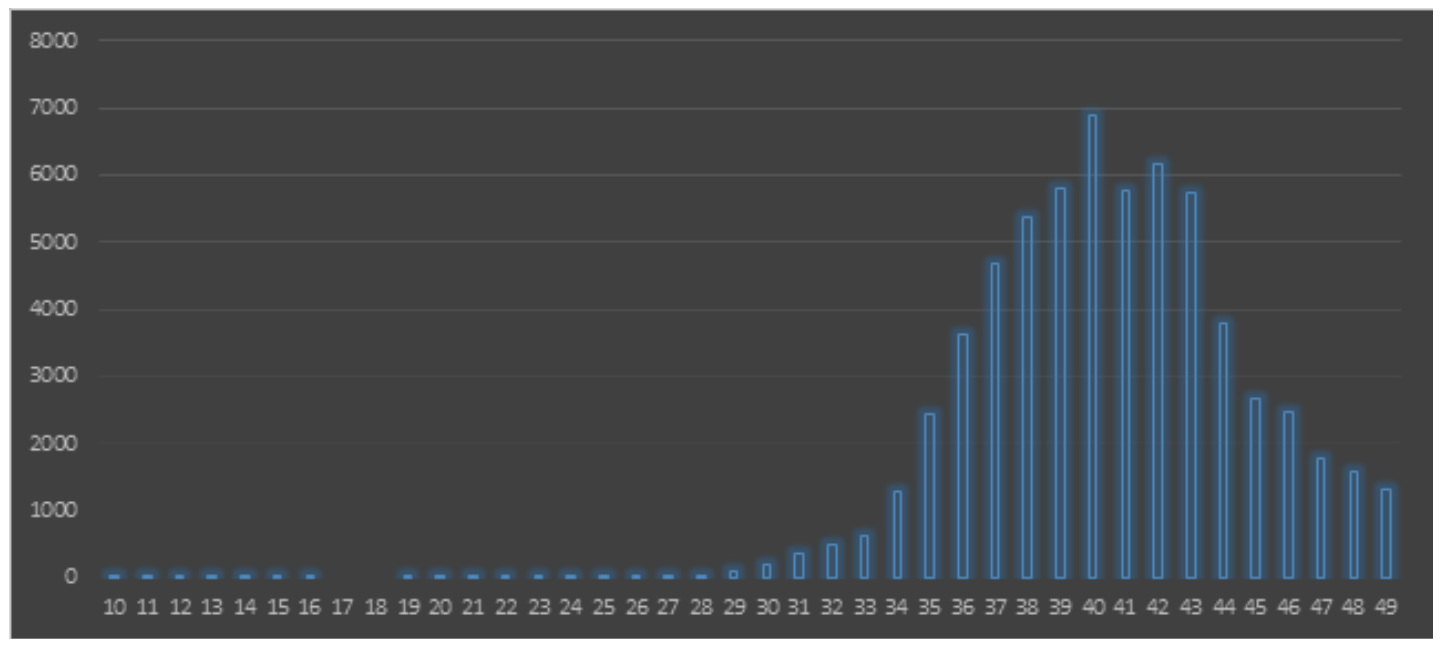

Figura 1: Casos confirmados COVID19 por semana epidemiológica (SE) en la ciudad de Rosario. Periodo: SE 10 (01/03/202007/03/2020) - 49 (29/11/2020—05/12/2020). (Fuente: Informes Situación COVID y Sala de Situación, MR).

\section{Primera etapa: preparación y desconocimiento}

Fue la de preparación de los equipos para la novedosa atención y seguimiento de casos COVID19. Esto implicó la capacitación de los trabajadores en relación a un cuadro clínico desconocido, con requerimiento de elementos de protección personal (EPP) especiales y modos de organización diferentes. En términos temporales, podríamos ubicar este período entre el 13/3/20 cuando se realizó la primera intervención preventiva poblacional y mediados de abril de 2020 (SE 12-18), cuando se constató un éxito parcial con el aplanamiento de la curva de casos. (Ver Figura 1) Esta primera etapa estuvo caracterizada por la incertidumbre y una compleja sensación de temor por el advenimiento de la catástrofe observada en Europa.

Los modos de presentación de los padecimientos se re configuraron en la pandemia. Problemas como la violencia contra la mujer mostraron tendencias en aumento o presentaciones más complejas. Las personas de los grupos de riesgo tenían que aislarse en sus domicilios lo que los exponía más aún a la soledad, las dificultades de acompañamiento y la necesidad de autocuidado.

El primer desafío de los equipos fue establecer una nueva modalidad de trabajo en el marco del ASPO. Se presentó la necesidad de suspender atenciones no urgentes y desarrollar modos alternativos. Por ejemplo, se implementaron campañas de vacunación con resultados destacables: entre 25/3 y 12/4 se colocaron 103.339 dosis de vacuna antigripal lo que implica un 80\% más que las aplicadas en el mismo periodo del 2019. 
Asimismo, este periodo estuvo caracterizado por la coexistencia de COVID y dengue. En 2020, la incidencia acumulada de casos de dengue en Rosario, alcanzó la categoría de epidemia. El 25/8/20 se habían reportado 1506 casos y 2 fallecimientos, la mayor incidencia se observó entre las semanas epidemiológicas 11 (8/3-14/3) y 20 (10/5-16/5). Esto implicó un desafío particular en la atención de personas con cuadros febriles dada la inédita coincidencia de ambas epidemias.

\section{Segunda etapa: organización de los equipos de salud}

En este momento, los equipos sanitarios ya estaban capacitados en el uso de EPP y en la atención de casos de COVID19. La mayoría de los CS establecieron dos circuitos diferenciados con un triage al ingreso de las instituciones: uno para casos sospechoso y otro para consultas espontáneas y seguimiento por problemas no relacionados a COVID. Este circuito incluyó atención de urgencias, seguimiento, vacunación y entrega de medicación a personas de riesgo, seguimiento de embarazadas y modos alternativos de atención integral de situaciones socio subjetivas, entre otras intervenciones. Esta etapa estuvo caracterizada por la baja demanda de atenciones por cuadros respiratorios en todas las edades, debido probablemente a la eficacia del ASPO, y a la organización de los equipos de CS para sostener prácticas de cuidados integral en las personas de mayor riesgo. Se podría establecer esta etapa entre mediados de abril 2020 y las primeras semanas de junio 2020. Este periodo se transitó con la amenaza siempre latente del advenimiento de un pico de contagios. Imágenes desgarradoras como las fosas comunes para muertos que se contaban de a miles en diferentes puntos del planeta establecieron fuerte impacto. Superando angustias y temores iniciales, los equipos de CS aumentaron la capacidad resolutiva, modificaron el proceso de trabajo y profundizaron la descentralización de los servicios de salud. Pero, emergieron desigualdades: los sectores más empobrecidos no tienen condiciones para sostener el ASPO dado que muchas personas viven del trabajo informal o contratos temporales. La presencia del Estado en los territorios más vulnerabilizados se circunscribió casi exclusivamente al sector salud. Entonces, los equipos de CS se constituyeron en instituciones receptoras de demandas heterogéneas: ayudas alimentarias, Asignaciones Familiares, Ingreso Familiar de Emergencia (IFE), prestaciones por discapacidad, problemas de cobertura del subsector de la Seguridad Social, situaciones de violencia, entre muchas otras. El personal que hacía triage al ingreso de cada CS se constituyó ineludiblemente en la primera, y a veces única, presencia del Estado en muchos barrios de la ciudad. En simultáneo, la demanda de atención en guardias de hospitales mostró un marcado descenso en el periodo comprendido entre marzo y agosto. (ver Figura 2)

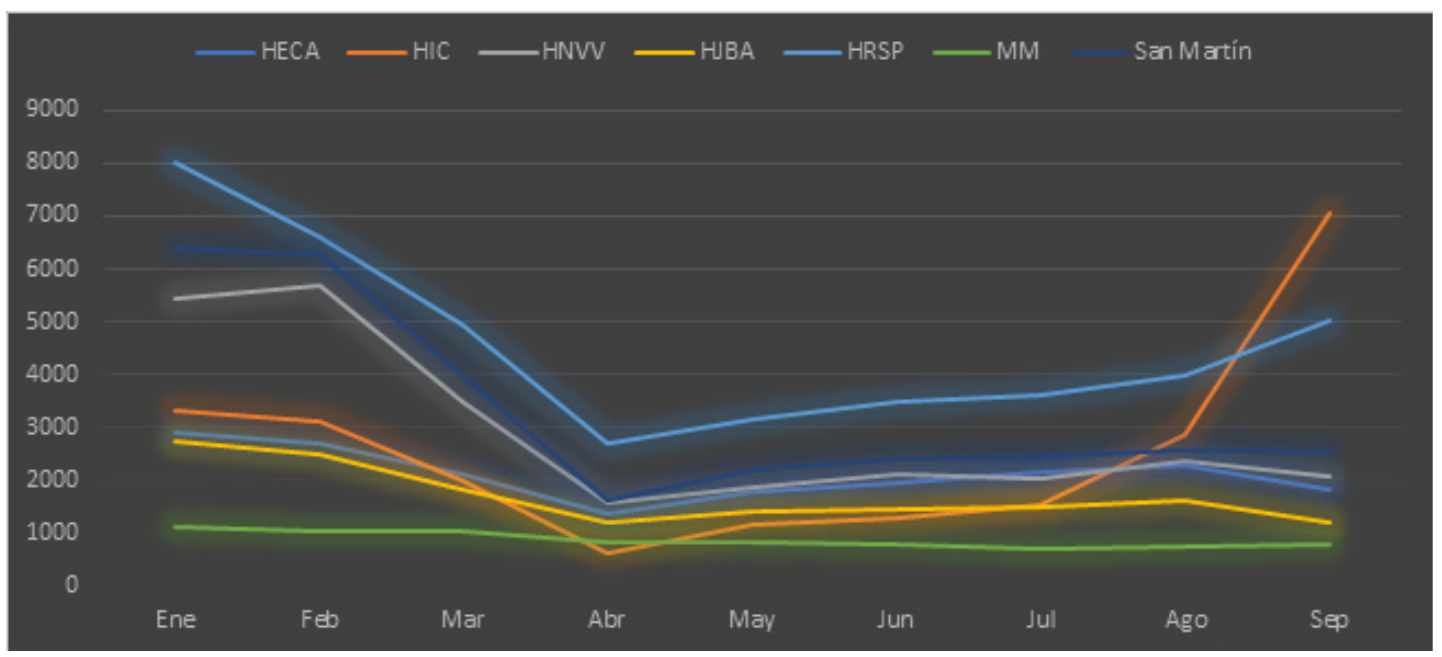

Figura 2: Tarjetas de guardia (registros de admisión) en hospitales dependientes de la SSP—MR del año 2020, según informe de Datos Abiertos de la Sala de Situación de la MR.

La Comisión Económica para América Latina y el Caribe (CEPAL) publicó una proyección que señalaba para la región una alarmante contracción de la actividad económica (—5.3\%) para el 2020. Se pronosticó la peor caída de la economía en Latinoamérica desde las crisis de 1914 y 1930. 
Apareció la tensión entre libertad o privilegio individual y cuidado colectivo. Algunas posturas negacionistas respecto a la dimensión y los riesgos de la pandemia surgían en diferentes puntos del planeta. En Europa, Suecia de la mano de su epidemiólogo de referencia A. Tegnell presentaba una de las estrategias más liberales del mundo. Se regulaba la vida social con mínimas restricciones, se manifestaba oposición al uso masivo de barbijos, se invitaba al auto reporte de síntomas como mecanismo de rastreo de casos y se utilizaron criterios muy flexibles de cuarentena de contactos para evitar el ausentismo laboral. Algunos medios locales, replicaban esta experiencia como ejemplo en contraste con el ASPO implementado en Argentina. En Norteamérica, su presidente D. Trump invitaba a no dejar de trabajar aun con cifras de contagios y muertes en el nivel de una catástrofe histórica. Incluso, se atrevió a manifestarse públicamente despectivo con el término "virus chino". En nuestra región, el presidente de Brasil, J. Bolsonaro, aportaba su cuota negacionista refiriéndose al virus como "gripeziñha" y montando una estrategia descoordinada, conflictiva, con pocas restricciones y, al igual que Trump, priorizando la economía por sobre el cuidado de la vida. En Argentina, aparecían las primeras convocatorias en oposición a las medidas de cuidado colectivo instauradas por el gobierno nacional.

\section{Tercera etapa: éxito parcial}

La ubicamos desde de junio hasta mediados de julio. Caracterizada por el advenimiento de un "valle" con equipos capacitados, alto grado de alerta para detectar casos y hospitales con bajo porcentaje de ocupación de camas. El 10/5/20 el Ministerio de Salud de Nación retiró a Rosario de la lista de ciudades con trasmisión comunitaria del virus. El 7/6/20 Santa Fe pasó a DISPO flexibilizando muchas de las restricciones de las etapas anteriores. La cotidianeidad de gran parte de la población se acercó a una "nueva normalidad". Rosario logró la anhelada supresión viral. ${ }^{\mathrm{E}}$

Los equipos de CS sentían la tensión de la demanda gatillada por problemas de salud diferentes del COVID. La necesidad de una nueva sociabilidad en el marco del DISPO se presentaba como el gran desafío. Los equipos de los hospitales empezaban a preguntarse cuál era su rol sin demanda masiva de pacientes respiratorios. Por otro lado, los equipos territoriales, más cercano a los problemas de los barrios de Rosario, percibían una creciente y heterogénea necesidad. El modelo de atención predominante de los CS pujaba por re configurarse acorde a la coyuntura: cuidado integral en el marco del DISPO. El dispositivo del consultorio parece una modalidad agotada en este periodo. La visita domiciliaria, las postas de vacunación y diferentes modos de trabajo territorial volvían a presentarse como intervenciones fundamentales. También, se incorporaron nuevas prácticas acorde a la necesidad sanitaria del momento: se realizan hisopados nasofaríngeos en CS para facilitar la detección. Esta intervención fue esencial, dado que la temprana identificación de los casos y el oportuno aislamiento de los contactos (potenciales nuevos casos) es una estrategia eficaz para interrumpir las cadenas de contagios. Además, en los barrios con condiciones poblacionales de vulnerabilidad se organizaron intervenciones de rastrillaje en el marco del Plan DetecAR ${ }^{\mathrm{F}}$. De ese modo, se realizan un gran número de hisopados y se aumenta la detección. En simultáneo, se instaló desde marzo un sistema telefónico para consultas ante síntomas de COVID y para programar controles en domicilio, incluso con realización de hisopados. Estas intervenciones sanitarias, posiblemente, fueron claves en complementariedad con el ASPO para lograr la supresión viral en Rosario.

\section{Cuarta etapa: se avecina la tormenta.}

A partir de julio (SE 28) los casos en clúster o burbujas familiares/afectivas se fueron haciendo progresivamente más numerosos hasta que, finalmente, Rosario volvió a ingresar a la lista de ciudades con trasmisión comunitaria. (ver gráfico 1) Velozmente, aparecen casos en todos los distritos, con

E Supresión: medidas de distanciamiento social generales con el objetivo de reducir el número reproductivo básico (R0) por debajo de 1 y, por lo tanto, reducir el número de casos a niveles bajos y/o eliminar la transmisión de persona a persona. (Ferguson, N., Laydon, D., Nedjati-Gilani, G., Imai, N., Ainslie, K., Baguelin, M., ... \& Dighe, A. (2020). Report 9: Impact of non—pharmaceutical interventions (NPIs) to reduce COVID19 mortality and healthcare demand. Imperial College London, 10, 77482.)

F Plan DetecAR: intervención coordinada entre los gobiernos Nacional, Provincial y Municipal para aumentar la capacidad de testeo y detección de casos. Se presentó su ampliación con test de detección rápida de antígenos que llevaría la capacidad a mil test diarios. (https://www.paginal2.com. ar/295476-alberto-fernandez-lanzo-en-rosario-el-plan-detect-ar-federal) 
amplio predominio del centro, el cual acumuló la mayor cantidad de casos activos. (Ver Figura 3) En este territorio, vive la población con mayor proporción de cobertura sanitaria a través de obras sociales y/o medicina privada. Por lo tanto, la estrategia de abordaje en el centro fue, probablemente, muy diferente que aquella implementada en los demás distritos de la ciudad.

Aunque las sugerencias no fueron tomadas en cuenta, científicos de CONICET alertaban sobre el riesgo inminente de aumento de casos y de pérdida de la oportunidad de control de la pandemia mediante los rastreos y aislamientos. En base a proyecciones de modelos matemáticos, recomiendan intervenciones poblacionales para disminuir el contacto social y frenar los contagios antes de que empeore el escenario. Detallaban que intervenir precozmente podría ser más eficaz.

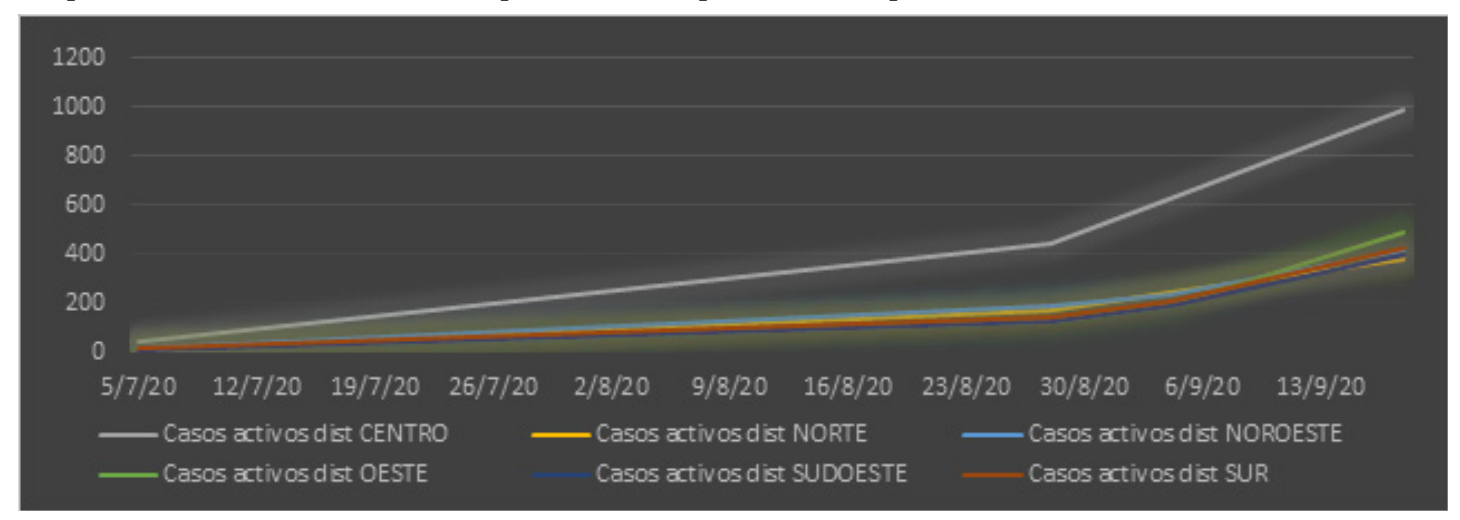

Figura 3: Casos activos por distrito de Rosario entre el 5/7/20 y el 18/9/20. (Fuente: Informes de Situación COVID. Municipalidad de Rosario).

Las camas de internación general y críticas de efectores públicos y privados empiezan a aumentar los porcentajes de ocupación. Las y los trabajadores de salud sienten un gran agotamiento relacionado a la carga subjetiva y a la demanda creciente de atención. Asimismo, en el marco de la Emergencia Sanitara las licencias continúan suspendidas. Por otro lado, emergen diferentes tipos de conflictos: trabajadores en reclamo por mejores condiciones y recomposición salarial, grupos opuestos a las medidas del gobierno nacional que salen a manifestarse con consignas dispares (anti vacunas, anti cuarentena, liberales, anti peronistas, etc.) y sectores cuya actividad laboral se vio fuertemente afectada por la pandemia como el turismo, gastronómicos, jardines, salones de eventos y espectáculos. En salud, la falta de paritarias en las administraciones municipales y provinciales, entre otros motivos, gatilla la instauración de medidas de fuerza propuestas por diferentes sindicatos.

En simultáneo, se mantiene la fase de DISPO con menos restricciones, pero la provincia instauró la prohibición de encuentros familiares y afectivos en el departamento Rosario a partir del 25/07/20. En el informe de situación COVID del 28/08/20 de la MR estableció un tiempo de duplicación de casos de 11 días y un porcentaje de ocupación de camas críticas de adultos del 50\% en efectores privados y del $75 \%$ en efectores públicos. Con estos indicadores, el escenario más temido se presentaba inminente. Sin embargo, importantes sectores de la población, amparados en las normas del DISPO, sostenían actividades sociales, deportivas y laborales que favorecían contagios. Los gobiernos nacional, provincial y municipal apelaban a la responsabilidad individual, aunque esto no se veía reflejado aún en los indicadores sanitarios. El discurso oficial destacaba, entre otras, la frase "aprender a convivir con el virus" que, tal vez, invitaba a la naturalización de las muertes. Se señalaba el porcentaje de ocupación de camas críticas, la tasa de letalidad y la afectación de grupos de riesgo como los indicadores clave para definir necesidad de implementar nuevas restricciones. El personal de salud padece el contraste entre servicios de salud tensionados y un mundo "fuera de salud" donde parece desconocerse la gravedad de la situación. A diferencia de lo observado en las primeras etapas, aun frente a números alarmantes las autoridades exponen señales de menor capacidad y/o convicción para instalar medidas poblaciones. Los discursos sobre el riesgo, la necesidad de cuidado colectivo y el sentido común de la sociedad parecen perder contundencia, y resurgen discursos en defensa de la libertad como privilegio individual aun a costa de la salud de otras personas. Las proyecciones alarmantes y las recomendaciones que realizaron científicos locales no fueron priorizadas en la toma de decisiones. Las difusas, agresivas y contradictorias manifestaciones de indignados permanecen en algunos sectores minoritarios, pero se replican magnificadas en medios de comunicación y redes sociales. 


\section{Quinta etapa: en medio de la crisis.}

Estuvo caracterizada por la tensión del sector salud con un aumento de la demanda relacionado con la creciente curva de casos y con las medidas poblacionales confusas y parciales. (Ver Figura 1: SE 36-42) Una nueva intervención desde el Ejecutivo provincial marca el cambio de etapa. El 4/9/20, el gobernador decreta por el lapso de 14 días la suspensión de todas las actividades comerciales, recreativas, educativas, gastronómicas, deportivas, de ocio y de servicios no indispensables en los departamentos Rosario, San Lorenzo, Caseros, Gral. López y Constitución. ${ }^{\text {G }}$ Sólo persistieron habilitadas las actividades definidas como esenciales. Si bien, inmediatamente surgieron sectores que manifestaron su desacuerdo, la medida fue acompañada por una proporción mayoritaria de la población. Ante el escenario potencial e inminente de colapso sanitario, la intervención es bienvenida por el personal de salud. Un leve enlentecimiento en la curva de ascenso de casos atestiguó un grado significativo de aceptación de las restricciones por parte de la ciudadanía.

Sin embargo, en un confuso discurso luego de los 14 días se definieron medidas de flexibilización que invitaron a la ciudadanía a retomar actividades. Así, se configuró un mensaje contradictorio: medidas poblacionales favorecedoras de contagios en contexto de pleno aumento de casos, fallecimientos y ocupación de camas críticas.

La necesidad de abordajes integrales para la pandemia gana difusión en el ambiente académico internacional de la mano de una editorial publicada en The Lancet donde se destaca la dimensión social de la enfermedad, retomando el concepto de sindemia de Merril Singer.

Equipos de gestión de diferentes áreas de salud pública municipal manifestaron masivamente su pedido de intervenciones generales para frenar la transmisión. Un grupo de trabajadores del sector salud constituían la "Asamblea de los Trabajadorxs por la Salud Colectiva” y demandaban a las autoridades un cambio de estrategia de afrontamiento de la pandemia, priorizando la idea de cuidado colectivo. Presentaban como inaceptable la proyección de muertes por COVID y tomaban propuestas de intervención como las que sugieren los científicos del CONICET: Aislamiento Selectivo, Planificado e Intermitente (ASPI). Se trata de una estrategia con periodos de cierres intercalados con fases de apertura. La selectividad sería regional, temporal y por actividades. La planificación ofrecería una agenda de intermitencia preestablecida para ofrecer un panorama de certeza a la ciudadanía y para la planificación de actividades laborales, productivas y recreativas. Esta opción alejaría la posibilidad de colapso sanitario. El pedio se sintetizaba en dos frases: "cerrar para no contagiar" y "abrir para trabajar”. La trazabilidad de los casos, amplia y eficazmente lograda en Rosario, podría volver a ser posible. Sin embargo, las decisiones requeridas para "enfriar" la pandemia no se tomaron, aun cuando el presidente de la nación decretó el 11/10/20 que Rosario era uno de los 18 departamentos que debían volver al ASPO. ${ }^{\mathrm{H}}$ El 13/10/20 los nuevos casos ascendieron a 1251 y el porcentaje de ocupación de camas críticas era de $87 \%$ en el sector público de la ciudad. La tasa de mortalidad por millón de habitantes en Rosario crecía de modo alarmante. (Ver Figura 4)

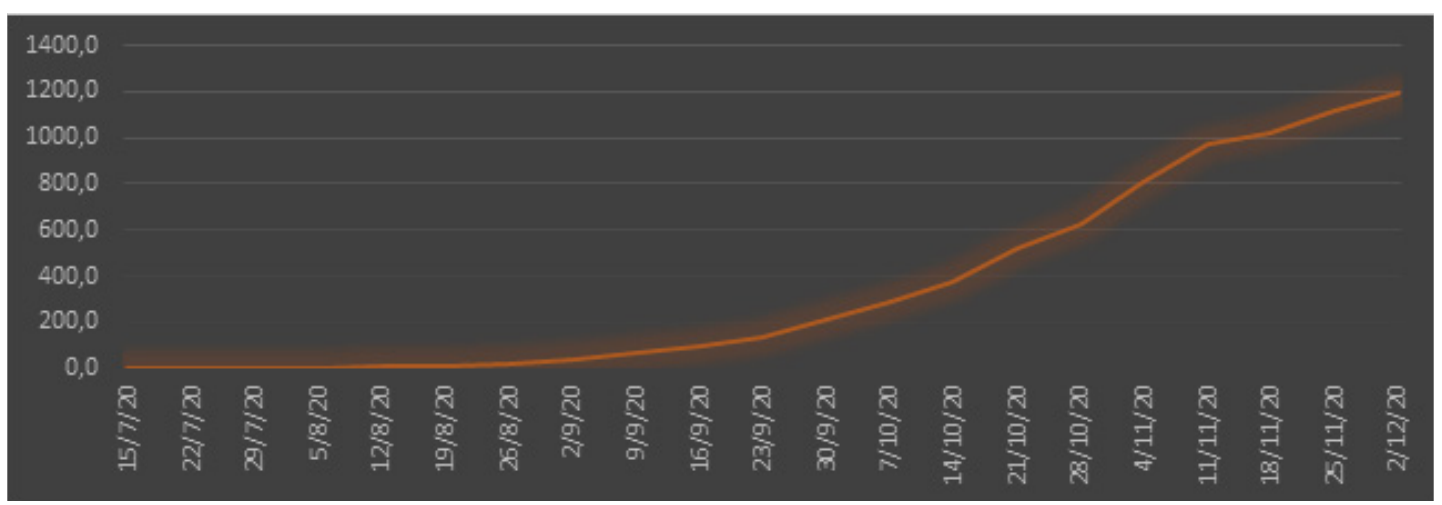

Figura 4: Tasa mortalidad por COVID19 por cada millón de habitantes de la ciudad de RosarioI, periodo: 15/7/20—02/12/20. (Calculada a partir de datos de Informes de Situación COVID, Municipalidad de Rosario).

G DECRETO N ${ }^{\circ}$ 0944, Provincia de Santa Fe. (4/9/20) Disponible en: https:/www.santafe.gob.ar/ms/covid19/wp—content/uploads/sites/36/2020/09/ D0094420.pdf

H AISLAMIENTO SOCIAL, PREVENTIVO Y OBLIGATORIO Y DISTANCIAMIENTO SOCIAL, PREVENTIVO Y OBLIGATORIO, Decreto 792/2020

(11/10/20) Disponible en: https://www.boletinoficial.gob.ar/detalleAviso/primera/235931/20201012 


\section{Sexta etapa: el descenso de la curva.}

La SE 40 (27/09/2020 - 03/10/2020) fue la de mayores casos confirmados hasta hoy y desde la SE 42 en adelante se observó un paulatino descenso de la curva. Sin embargo, dicho decrecimiento muestra un enlentecimiento en su velocidad de caída. La apertura prematura y poco cuidada de actividades podría haber sido un factor relacionado al descenso lento y "amesetamiento" de la curva con perpetuación de los contagios (ver Figura 1: SE 45-49). A su vez, las definiciones de aperturas de actividades y las tendencias en baja de los casos nuevos, probablemente, invitaron a la ciudadanía a un peligroso relajamiento de los cuidados. En esta etapa, la noción del cuidado colectivo parece perder vigencia en el sentido común. El escenario más temido del colapso del sistema de salud se aleja en las posibilidades inmediatas. Autoridades planteaban la hipótesis de que los casos bajan debido a la incorporación de los protocolos sanitarios, la adopción de medidas de cuidado individual y las condiciones climáticas estivales, pero se alertaban sobre las potenciales consecuencias del relajamiento. Por otro lado, la llegada de vacunas para iniciar campañas masivas ingresaba fuertemente en la agenda pública y abonaba a la falsa idea de un inminente fin de pandemia. La muerte de Diego Maradona, su funeral y todas las repercusiones del mismo fueron replicados en los medios de comunicación como elemento para criticar las medidas del gobierno nacional.

Rosario acumulaba al 02/12/20 la preocupante cifra de 1192 muertes reportadas por COVID19. En proyección de las fiestas de fin de año, la amenaza de un posible re brote, las alarmantes tasas de muertes por millón de habitantes y las potenciales consecuencias a largo plazo del COVID no parecían ser suficientes para volver a posicionar al cuidado en el centro de la escena. Un clima de "lo peor ya pasó" impresionaba fuertemente instalado, la igual que la naturalización de las muertes y la postergación de la necesidad individual y colectiva del cuidado.

\section{Reflexiones finales y algunas preguntas}

Esta crónica de la pandemia en la ciudad de Rosario, ofrece perspectivas para plantear preguntas.

La ciudad de Rosario logró la supresión de la transmisión viral prácticamente al mes de implementación del ASPO. El acompañamiento de la mayor parte de la población hizo efectiva esta medida. Sin embargo, a partir de julio, la ciudad pasó de casos en burbujas a una circulación comunitaria en pleno aumento. Casi la totalidad de los casos se relacionaban con viajes interprovinciales y la falta de cuidado por parte personas que no quisieron o no pudieron tomar las precauciones pertinentes. ¿Qué estrategias podrían haber diseñado los gobiernos por intervenir en este periodo? Los análisis matemáticos de científicos de CONICET sugerían que otro camino era posible.

La comunidad científica internacional reconoce la eficacia y necesidad de medidas no farmacológicos como el distanciamiento social y el rastreo/aislamiento de casos y contactos. Los reportes epidemiológicos sugieren que, en Rosario, el ASPO fue efectivo para suprimir la circulación viral y para la preparación de los servicios de salud. Sin embargo, también fue observado que disparó debates, controversias y resistencia. En perspectiva del advenimiento de un posible re brote, no parece una medida aplicable a largo plazo o por periodos prolongados. Propuestas diferentes como el ASPI podrían ser estrategias más convocantes, sustentables y superadores.

Además, los indicadores de ocupación de camas hospitalarias impresionaron ser el parámetro más relevante al momento de definir medidas para disminuir contacto social y contagios. Observando el número de muertes por COVID19 en Rosario, parece prioritario reflexionar si las curvas de casos confirmados debieran ser el indicador fundamental para definir la implementación de medidas no farmacológicas. Probablemente, el aumento de ocupación de camas críticas sea un parámetro tardío para definir las intervenciones.

Respecto de la distribución territorial, el distrito centro fue el que registró más casos. La respuesta sanitaria implementada allí fue, posiblemente, diferente a la realizada en el resto de la ciudad. En el distrito centro, se delegó la tarea de rastreo/aislamiento al sub sector privado y de la seguridad social. Se identifica la necesidad de investigar lo transitado y revisar la estrategia de rastreo/aislamiento de casos y contactos del distrito centro en los próximos meses.

A su vez, el ASPO desnudó las desigualdades de la sociedad, perpetuamente invisibilizadas. Se presenta la forzosa urgencia de re pensar la presencia del Estado en muchos barrios de la ciudad de Rosario para acompañar y mitigar el impacto multidimensional de la pandemia y en simultáneo, para detectar, diagnosticar y apoyar el aislamiento de los casos y sus contactos.

Por otro lado, la perspectiva de las autoridades parece enfocarse en la anhelada post pandemia y en 
la solución a partir de intervenciones biomédicas como la vacuna. Sin embargo, atravesar la pandemia será un proceso dinámico y de duración indeterminada aún. Las vacunas no aportarán una solución inmediata. Frente a este escenario, urge diseñar y explicitar las estrategias de afrontamiento de la pandemia en los meses venideros.

Resulta ineludible revisar el discurso dominante sobre la pandemia. "Convivir con el virus" sugiere el acercamiento a una estrategia de inmunidad de rebaño por vía natural y una peligrosa naturalización de las muertes. Elllamado a la responsabilidad individual como convocatoria principal podría suponer una transferencia de responsabilidades de los gobiernos a las personas. Superar la negación que la crisis desencadena, enfrentarnos con la angustia de un cotidiano complejo e incierto, podría ser un camino para desnaturalizar los fallecimientos y volver a convocar al cuidado colectivo.

Fuentes de financiamiento: no presenta.

\section{Referencias bibliográficas}

1. Cascella, M., Rajnik, M., Cuomo, et al (2020). Features, evaluation and treatment coronavirus (COVID-19). In Statpearls [internet]. StatPearls Publishing.

2. Cheng VC, Lau SK, Woo PC, et al. Severe acute respiratory syndrome coronavirus as an agent of emerging and reemerging infection. Clin Microbiol Rev. 2007;20(4):660-694.

3. Chan JF, Lau SK, To KK, et al. Middle East respiratory syndrome coronavirus: another zoonotic betacoronavirus causing SARS-like disease. Clin Microbiol Rev. 2015;28(2):465-522.

4. Huang C, Wang Y, Li X, et al. Clinical features of patients infected with 2019 novel coronavirus in Wuhan, China. Lancet. 2020 Feb 15;395(10223):497-506.

5. World Health Organization. Coronavirus disease (COVID-2019): situation report 72 [Internet].

6. Genebra: World Health Organization; 2020. Disponible en: https://www.who.int/docs/default-source/coronaviruse/situation-reports/20200401sitrep-72-covid-19.pdf?sfvrsn=3dd8971b_2

7. Li, Q., Guan, X., Wu, et al. Early transmission dynamics in Wuhan, China, of novel coronavirus-infected pneumonia. N Engl J Med 2020; 382:1199-1207

8. Lam, S., Lombardi, A., Ouanounou, A. COVID-19: A review of the proposed pharmacological treatments. European journal of pharmacology $2020,886,173451$

9. McCreary EK, Pogue JM. Coronavirus Disease 2019 Treatment: A Review of Early and Emerging Options. Open Forum Infect Dis. 2020 Mar 23;7(4):ofaal05

10. Bhimraj A, Morgan RL, Shumaker AH, et al. Infectious Diseases Society of America Guidelines on the Treatment and Management of Patients with COVID-19. Clin Infect Dis. 2020 Apr 27:ciaa478.

11. Ministerio de Salud de la Argentina. Recomendaciones condicionales para el abordaje terapéutico de COVID-19 - Versión 3.0 (septiembre 2020). Disponible en: https://www.argentina.gob.ar/salud/coronavirus-COVID-19/abordaje-terapeutico

12. RECOVERY Collaborative Group, Horby P, Lim WS, Emberson JR, et al. Dexamethasone in Hospitalized Patients with Covid-19 - Preliminary Report. N Engl J Med. 2020 Jul 17:NEJMoa2021436.

13. Hodgson SH, Mansatta K, Mallett G. What defines an efficacious COVID-19 vaccine? A review of the challenges assessing the clinical efficacy of vaccines against SARS-CoV-2. Lancet Infect Dis. 2021 Feb;21(2):e26-e35.

14. Dong Y, Dai T, Wei Y, et al. A systematic review of SARS-CoV-2 vaccine candidates. Signal transduction and targeted therapy 2020, 5(1), 1-14.

15. Heaton, P. M. (2020). The Covid-19 vaccine-development multiverse. N Engl J Med 2020; 383:1986-1988

16. Nature. Long COVID: let patients help define long-lasting COVID symptoms. Nature 2020; 586: 170.

17. Carfi, A., Bernabei, R., Landi, F. Persistent symptoms in patients after acute COVID-19. Jama 2020, 324(6), 603-605.

18. Perego E, Callard F, Stras L et al. Why the Patient-Made Term 'Long Covid' is needed [version 1; peer review: 1 approved with reservations, 1 not approved]. Wellcome Open Res 2020, 5:224

19. José RJ, Manuel A, Gibson-Bailey K, Lee L. Post COVID-19 bronchiectasis: a potential epidemic within a pandemic. Expert Rev Respir Med. 2020 Dec;14(12):1183-1184.

20. Huang L, Zhao P, Tang D, et al. Cardiac Involvement in Patients Recovered From COVID-2019 Identified Using Magnetic Resonance Imaging. JACC Cardiovasc Imaging. 2020 Nov;13(11):2330-2339.

21. Mitrani RD, Dabas N, Goldberger JJ. COVID-19 cardiac injury: Implications for long-term surveillance and outcomes in survivors. Heart Rhythm. 2020 Nov;17(11):1984-1990.

22. Lahiri D, Ardila A. COVID-19 Pandemic: A Neurological Perspective. Cureus. 2020;12(4):e7889.

23. Ferguson N, Laydon D, Nedjati-Gilani G, et al. Report 9: Impact of non-pharmaceutical interventions (NPIs) to reduce COVID19 mortality and healthcare demand. Imperial College London 2020, 10, 77482.

24. Flaxman S, Mishra S, Gandy A, et al. Estimating the effects of non-pharmaceutical interventions on COVID-19 in Europe. Nature 2020, 584(7820), 257-261.

25. Alwan NA, Burgess RA, Ashworth S, et al. Scientific consensus on the COVID-19 pandemic: we need to act now [published correction appears in Lancet. 2020 Oct 19;:]. Lancet. 2020;396(10260):e71-e72. 
Sochas L, Channon AA, Nam S. Counting indirect crisis-related deaths in the context of a low-resilience Health system: the case of maternal and neonatal health during the Ebola epidemic in Sierra Leone. Health Policy Plan 2017; 32(suppl_3): iii32-iii39

30. Ministerio de Salud, Prov. Santa Fe, 2020. En: https://www.santafe.gov.ar/index.php/web/content/view/full/114837/(subtema)/114560 (Consultado: 01/03/2020)

31. Secretaría Salud Pública, Municipalidad de Rosario, 2020. En: https://www.rosario.gob.ar/web/servicios/salud/hospitales/ (Consultado: 01/03/2020)

32. Rovere, M. (2005). "Una ciudad modelo en salud pública. Aportes a la construcción de una gobernabilidad democrática. Experiencia Rosario. Políticas para la gobernabilidad.” En: https://www.elagora.org.ar/site/documentos/Experiencia_Salud_Rosario.pdf

33. Merhy, E. E., \& De Vega, L. I. N. (2006). Salud: cartografía del trabajo vivo. Lugar Editorial.

34. Guan WJ, Ni ZY, Hu Y, et al. Clinical characteristics of coronavirus disease 2019 in China. New England journal of medicine 2020, 382(18), 1708-1720.

35. Thevarajan I, Buising KL, Cowie BC. Clinical presentation and management of COVID-19. Med J Aust. 2020 Aug;213(3):134-139.

36. Rovere, M. (2018). La atención primaria en la encrucijada: desplegar su potencial transformador o quedar instrumentados en una nueva ofensiva privatizadora. Saúde em Debate, 42, 315-327.

37. Informes de Situación COVID. Municipalidad de Rosario. Disponibles en https://www.rosario.gob.ar/web/coronavirus/coronavirus-informesde-situacion-y-tableros)

38. PANEL DE CONTROL: Coronavirus - CIUDAD DE ROSARIO - AÑO 2020. COVID-19, Sala de situación, Municipalidad de Rosario. Disponible en: https://www.rosario.gob.ar/mr/epidemiologia/sala-de-situacion/curvas-epidemicas-y-canales-endemicos/coronavirus/coronavirus-ano-2020/ coronavirus

39. Italia registró 766 muertes por coronavirus en las últimas 24 horas y sumó 4.585 contagios (Nota periodística, 03/04/20). Disponible en: https:// www.infobae.com/america/mundo/2020/04/03/italia-registro-766-muertes-por-coronavirus-en-las-ultimas-24-horas-y-sumo-4585-contagios/

40. Coronavirus: España está “cerca de doblegar la curva” de la pandemia de covid-19 (Nota periodística, 04/4/20). Disponible en: https://www.bbc. com/mundo/noticias-internacional-52169654

41. Los femicidios y la violencia machista en cuarentena: la curva en ascenso (Nota periodística, 24/4/20). Disponible en: https://www.paginal2.com. ar/261481-los-femicidios-y-la-violencia-machista-en-cuarentena-la-curv

42. Violencia de género en cuarentena: aumentaron los pedidos de ayuda y faltan refugios que podrían evitar femicidios (Nota periodística, 30/3/20). Disponible en: https://www.infobae.com/sociedad/2020/03/30/violencia-de-genero-en-cuarentena-aumentaron-los-pedidos-de-ayuda-yfaltan-refugios-que-podrian-evitar-femicidios/

43. Entrevista a Soledad Guerrero, Coordinadora del Programa de Inmunizaciones, Prov. Santa Fe. (Nota Periodística, 14/4/20). Disponible en: https:// www.rosarioplus.com/en-otros-temas/salud/mas-de-cien-mil-rosarinos-ya-recibieron-la-vacuna-antigripal-_a5f4ab28el2b5372badfb8712

44. Informe 30/10/20. Fuente: SICAP Sistema Información Centro de Atención Primaria. PROGRAMA PROVINCIAL DE INMUNIZACIONES. DIRECCIÓN PROVINCIAL DE PROMOCION Y PREVENCION DE LA SALUD. Ministerio Salud, Prov. Santa Fe.

45. Informe de situación de dengue Ciudad de Rosario - Año 2020 (25 de agosto de 2020) Disponible en: https://www.rosario.gob.ar/mr/epidemiologia/ sala-de-situacion/curvas-epidemicas-y-canales-endemicos/sindrome-febril/dengue-1/dengue-ano-2020/informe-dengue-2020/view

46. Coronavirus en Brasil: Manaos, la ciudad azotada por la pandemia que cava fosas comunes y reclama ayuda (Nota Periodística, 4/5/20). Disponible en: https://www.bbc.com/mundo/noticias-america-latina-52530718

47. Abren fosas comunes en una isla de Nueva York (Nota periodística, 11/4/20). Disponible en: https://www.paginal2.com.ar/258901-abren-fosascomunes-en-una-isla-de-nueva-york

48. Detrás del coronavirus, la desigualdad y otras pandemias acechan desde hace más tiempo (Nota periodística, 30/5/20) Disponible en: https:// www.elciudadanoweb.com/detras-del-coronavirus-la-desigualdad-y-otras-pandemias-acechan-desde-hace-mas-tiempo/

49. Coronavirus, desigualdad y neoliberalismo: la falsa idea de que la pandemia ataca a todos igual tiempo (Nota periodística, 16/5/20) Disponible en: https://www.elciudadanoweb.com/coronavirus-desigualdad-y-neoliberalismo-la-falsa-idea-de-que-la-pandemia-ataca-a-todos-igual/

50. Sala de Situación. Municipalidad de Rosario. (consulta 10/10/20) RosarioDatos (datos abiertos). Disponible en: https://www.rosario.gob.ar/mr/ epidemiologia/sala-de-situacion/rosariodatos-datos-abiertos

51. Pandemia del COVID-19 llevará a la mayor contracción de la actividad económica en la historia de la región: caerá -5,3\% en 2020. Comunicado de Prensa CEPAL (24/04/20). Disponible en: https://www.cepal.org/es/comunicados/pandemia-covid-19-llevara-la-mayor-contraccion-la-actividadeconomica-la-historia-la

52. Gretchen V. Sweden's gamble. Science 2020, 370 (6513), 159-163.

53. El modelo de Suecia para ganarle al coronavirus. (Nota periodística, 30/04/20) Disponible en: https://www.infobae.com/america/the-new-yorktimes/2020/04/30/suecia-esta-haciendo-lo-correcto/

54. April Was Trump's Cruelest Month (Nota periodística, 27/08/20) Disponible en: https://www.nytimes.com/2020/08/27/opinion/donald-trumpcoronavirus.html

55. Trump se refirió al "virus chino" y desató la furia en Beijing. (Nota periodística, 17/03/20) Disponible en: https://www.cronista.com/ internacionales/Trump-se-refirio-al-virus-chino-y-desato-la-furia-en-Beijing-20200317-0009.html 
56. Desde "gripezinha" a "la vida continúa": las frases con las que Jair Bolsonaro minimizó el coronavirus. (Nota periodística, 07/07/20) Disponible en: https://www.clarin.com/mundo/gripezinha-vida-continua-frases-jair-bolsonaro-minimizo-coronavirus_0_aVXRIiYBn.html

57. La estrategia de Bolsonaro contra el coronavirus lo enfrenta al Ejército y a los gobernadores. (Nota periodística, 26/03/20) Disponible en: https:// elpais.com/sociedad/2020-03-27/la-estrategia-de-bolsonaro-contra-el-coronavirus-lo-enfrenta-al-ejercito-y-a-los-gobernadores.html

58. Convocan a una marcha en todo el país contra la cuarentena: reclaman "libertad para trabajar y movilizarse" (Nota periodística, 29/5/20) Disponible en: https://www.clarin.com/politica/convocan-marcha-pais-cuarentena-reclaman-libertad-trabajar-movilizarse-_0_t021LcAzA.html

59. Convocan a una marcha en todo el país contra la cuarentena (Nota periodística, 29/5/20) Disponible en:

60. https://www.ellitoral.com/index.php/id_um/241983-convocan-a-una-marcha-en-todo-el-pais-contra-la-cuarentena-en-redes-socialesnacionales.html

61. Ferguson N, Laydon D, Nedjati-Gilani G, et al. Report 9: Impact of non-pharmaceutical interventions (NPIs) to reduce COVID19 mortality and healthcare demand. Imperial College London 2020, 10, 77482.

62. Aleta A, Martín-Corral D, Piontti APY, Ajelli M, Litvinova M, Chinazzi M, Dean NE, Halloran ME, Longini IM Jr, Merler S, Pentland A, Vespignani A, Moro E, Moreno Y. Modeling the impact of social distancing, testing, contact tracing and household quarantine on second-wave scenarios of the COVID-19 epidemic. medRxiv [Preprint]. 2020 May 18:2020.05.06.20092841.

63. Plan DETECTAR. Gobierno de la Provincia de Santa Fe. Disponible en: https://www.santafe.gob.ar/ms/covid19/plan-detectar/ (Acceso: 30/11/20)

64. Coronavirus. Gobierno de la Provincia de Santa Fe. Disponible en: https://www.santafe.gob.ar/ms/covid19/(Acceso: 30/11/20)

65. Zonas definidas con transmisión comunitaria en Argentina (22/7/20). Disponible en: https://www.argentina.gob.ar/salud/coronavirusCOVID-19/clasificacion

66. Volver o no volver a la cuarentena: análisis de la propagación del covid en Rosario al 21 de julio. (Nota periodística, 25/7/20) Disponible en: https:// www.elciudadanoweb.com/volver-o-no-volver-a-la-cuarentena-analisis-de-la-propagacion-del-covid-en-rosario-al-21-de-julio/

67. Informes de Situación COVID. Municipalidad de Rosario. Disponibles en https://www.rosario.gob.ar/web/coronavirus/coronavirus-informesde-situacion-y-tableros)

68. Paritarias en cuarentena: el rompecabezas salarial (Nota periodística, 30/8/20) Disponible en: https://www.lacapital.com.ar/economia/ paritarias-cuarentena-el-rompecabezas-salarial-n2606271.html

69. El banderazo de protesta contra el Gobierno se extendió por el interior del país (Nota periodística, 17/8/20) Disponible en: https://www.lanacion. com.ar/politica/el-banderazo-protesta-gobierno-se-extendio-interior-nid2423781

70. Anticuarentenas, anti reformas, pro cloroquina y pro Vicentin: todo en el \#17a(Nota periodística, 18/8/20) Disponibleen: https://www.rosarioplus com/en-saco-y-corbata/politica/anticuarentenas--anti-reformas--pro-cloroquina-y-pro-vicentin--todo-en-el--17a_a5f4aaea412b5372badfb33fo

71. Tras la caída de Johnny B Good, el sector gastronómico pide extender horarios y asistencia (Nota periodística, 20/8/20) Disponible en: https:// www.elciudadanoweb.com/tras-la-caida-de-johnny-b-good-el-sector-gastronomico-pide-extender-horarios-y-asistencia/

72. "De duelo": jardines maternales piden protocolo para volver a abrir (Nota periodística, 06/8/20) Disponible en: https://www.rosarioplus.com/ covid-19/-de-duelo---jardines-maternales-piden-protocolo-para-volver-a-abrir_a5f4aaee012b5372badfb3aec

73. Los profesionales nucleados en Siprus volvieron a la calle para reforzar sus reclamos (Nota periodística, 20/8/20) Disponible en: https://www. lacapital.com.ar/la-ciudad/los-profesionales-nucleados-siprus-volvieron-la-calle-reforzar-sus-reclamos-n2604522.html

74. Los trabajadores municipales van a un paro de dos días la semana próxima (Nota periodística, 19/8/20) Disponible en: https://www.lacapital.com. ar/la-ciudad/los-trabajadores-municipales-van-un-paro-dos-dias-la-semana-proxima-n2604345.html

75. COVID-19: LA PROVINCIA SUSPENDE LAS REUNIONES FAMILIARES Y AFECTIVAS EN LAS LOCALIDADES DEL DEPARTAMENTO ROSARIO (23/07/20) Disponible en: https://www.santafe.gob.ar/noticias/noticia/268038/

76. SituaciónCovid-19, 28 de agosto de 2020, Municipalidad de Rosario. Disponible en: https:/www.rosario.gob.ar/web/sites/default/files/informe_ coronavirus_28-08-2020_sala_situacion.pdf

77. La provincia apela a la responsabilidad individual para evitar contagios de covid (Nota periodística, 5/8/20) Disponible en: https://www.rosario3.com/ informaciongeneral/La-provincia-apela-a-la-responsabilidad-individual-para-evitar-contagios-de-covid-20200805-0059.html

78. Javkin: "Más del 30\% de los contagios de Covid-19 se dan en las reuniones familiares" (Nota periodística, 17/7/20) Disponible en: https://www. lacapital.com.ar/la-ciudad/javkin-mas-del-30-los-contagios-covid-19-se-dan-las-reuniones-familiares-n2597769.html

79. Perotti aseguró que el Estado está avanzando en aspectos clave en la lucha contra el coronavirus (Nota periodística, 10/9/20) Disponible en: https://www. lacapital.com.ar/pandemia/perotti-aseguro-que-el-estado-esta-avanzando-aspectos-clave-la-lucha-contra-el-coronavirus-n2608947.html

80. MARTORANO: “ESTAMOS EN ALERTA PARA NO RETROCEDER DE FASE” (14/08/20) Disponible en: https://www.santafe.gob.ar/noticias/ noticia/268259/

81. Los anticuarentena no son sólo irresponsables: disputan el sentido de la palabra libertad (Nota periodística, 25/10/20) Disponible en: https:// www.elciudadanoweb.com/los-anticuarentena-no-son-solo-irresponsables-disputan-el-sentido-de-la-palabra-libertad/

82. La profecía que nadie quiso ver (Nota periodística, 25/10/20) Disponible en: https://www.paginal2.com.ar/301341-la-profecia-que-nadie-quiso-ver

83. Marchas anticuarentena: Con más cámaras que asistentes (Nota periodística, 14/9/20) Disponible en: https://www.paginal2.com.ar/291809marchas-anticuarentena-con-mas-camaras-que-asistentes

84. Kofman: "Disminuir el contacto social en Rosario ya evitó 3.400 contagios" (Nota periodística, 29/9/20) Disponible en: https://www. elciudadanoweb.com/kofman-disminuir-el-contacto-social-en-rosario-ya-evito-3-400-contagios/

85. Nueva estrategia por dos semanas (Nota periodística, 25/9/20) Disponibleen: https://www.paginal2.com.ar/294544-nueva-estrategia-por-dos-semanas 
86. Cuarentena en Rosario: una posible contradicción en el anuncio que hace ruido (Nota periodística, 24/09/20) Disponible en: https://www. rosario3.com/informaciongeneral/Cuarentena-en-Rosario-una-posible-contradiccion-que-hace-ruido-20200924-0090.html

87. Horton, R. (2020). Offline: COVID-19 is not a pandemic. Lancet (London, England), 396(10255), 874

88. Directores de centros de salud a Javkin y Perotti: "La falta de aislamiento desprotege a los de menos recursos" (Nota periodística, 2/10/20) Disponible en: https://www.rosario3.com/informaciongeneral/Directores-de-centros-de-salud-a-Javkin-y-Perotti-La-falta-de-aislamientodesprotege-a-los-de-menos-recursos-20201001-0068.html

89. El cuidado colectivo va a la cola (Nota periodística, 2/10/20) Disponible en: https://www.paginal2.com.ar/295881-el-cuidado-colectivo-va-a-la-cola

90. Profesionales de la salud coinciden en que hay que restringir actividades (Nota periodística, 19/10/20) Disponible en: https://www.lacapital.com. ar/la-ciudad/profesionales-la-salud-coinciden-que-hay-que-restringir-actividades-n2617691.html

91. Trabajadores de la salud: "No queremos ser testigos de muertes que son evitables" (Nota periodística, 12/10/20) Disponible en: https://www. elciudadanoweb.com/trabajadores-de-la-salud-no-queremos-ser-testigos-de-muertes-que-son-evitables/

92. Botón rojo y aislamiento intermitente. Ernesto Kofman (Encuentro Virtual, 17/10/20) Disponible en: https://www.youtube.com/ watch?v=Oex15mhvzQg\&feature=youtu.be

93. Botón rojo y aislamiento intermitente. Rodrigo Castro (Encuentro Virtual, 17/10/20) Disponible en: https://www.youtube.com/ watch?v=jOM61IekQ30\&feature=youtu.be

94. Un llamado para que el Estado sostenga el aislamiento y ayude a los más vulnerables (Nota periodística, 12/8/20) Disponible en: https://www. paginal2.com.ar/284430-un-llamado-para-que-el-estado-sostenga-el-aislamiento-y-ayud

95. Dr. Rodrigo Castro: “Aislamiento, selectivo, planificado e intermitente es nuestra propuesta" (Nota periodística, 26/09/20) Disponible en: https:// www.youtube.com/watch?v=VK_w5lnfpWO

96. Kofman describió los escenarios posibles de pandemia en Rosario y analizó cómo bajar los casos. (Nota periodística, 27/10/20) Disponible en: https:// www.elciudadanoweb.com/kofman-describio-los-escenarios-posibles-de-pandemia-en-rosario-y-analizo-como-bajar-los-casos/

97. Acción urgente en el Monumento (Nota periodística, 23/09/20) Disponible en: https://www.paginal2.com.ar/301041-accion-urgente-en-el-monumento

98. Protesta de personal de Salud en el Monumento: “Naturalizamos que cada día mueran entre 10 y 20 personas" (Nota periodística, 23/09/20) Disponible en: https://www.lacapital.com.ar/la-ciudad/protesta-personal-salud-el-monumento-naturalizamos-que-cada-dia-mueran-10-y-20personas-n2618579.html

99. Cuarentena en el interior: varias provincias evitan cerrar todo e interpretan "a su manera" el último decreto presidencial (Nota periodística, 13/10/20) Disponible en: https://www.clarin.com/politica/cuarentena-interior-varias-provincias-evitan-cerrar-interpretan-manera-ultimodecreto-presidencial_0_0FiB2GiB7.html

100. Tablero de situación COVID-19. Municipalidad de Rosario. (13/10/20) Disponible en: https://www.rosario.gob.ar/web/sites/default/files/ situacion_covid-19_2020-10-13.pdf

101. Informes de Situación COVID. Municipalidad de Rosario. Disponibles en https://www.rosario.gob.ar/web/coronavirus/coronavirus-informesde-situacion-y-tableros

102. Coronavirus: Informe municipal muestra el amesetamiento de la curva de contagios en Rosario (Nota periodística, 31/10/20) Disponible en: https://www.elciudadanoweb.com/coronavirus-informe-municipal-muestra-el-amesetamiento-de-la-curva-de-contagios-en-rosario/

103. La tasa de letalidad de Covid se mide mal, la carga de fallecidos está muy retrasada (Nota periodística, 08/12/20) Disponible en: https://www. lacapital.com.ar/la-ciudad/la-tasa-letalidad-covid-se-mide-mal-la-carga-fallecidos-esta-muy-retrasada-n2628811.html

104. Corremos un alto riesgo de que aumenten los casos sobre fin de año (Nota periodística, 20/11/20) Disponible en: https://www.rosarioplus.com/ en-otros-temas/salud/-corremos-un-alto-riesgo-de-que-aumenten-los-casos-sobre-fin-de-ano-_a5fb7f3a7d7b1263790ff4314

105. Médicos advierten sobre el riesgo de viajes y colonias de vacaciones en plena pandemia (Nota periodística, 28/11/20) Disponible en: https:// conlagentenoticias.com/medicos-advierten-sobre-el-riesgo-de-viajes-y-colonias-de-vacaciones-en-plena-pandemia/

106. Coronavirus: Caruana pide prepararse para el verano "sin perder de vista lo que pasa en Europa" (Nota periodística, 23/11/20) Disponible en: https://www.lacapital.com.ar/pandemia/coronavirus-caruana-pide-prepararse-el-verano-sin-perder-vista-lo-que-pasa-europa-n2625398.html

107. Coronavirus en Rosario: las razones de la caída de los casos positivos (Nota periodística, 28/11/20) Disponible en: https://www.rosario3.com/ informaciongeneral/Coronavirus-en-Rosario-las-razones-de-la-caida-de-los-casos-positivos-20201127-0074.html

108. Los detalles sobre las vacunas que podrían aplicarse en el país (Nota periodística, 12/12/20) Disponible en: https://www.telam.com.ar/ notas/202012/538078-vacunas-coronavirus-argentina.html

109. Cuándo empieza a aplicarse la vacuna contra el coronavirus en Argentina (Nota periodística, 24/11/20) Disponible en: https://www.clarin.com/ politica/ahora-gobierno-asegura-podria-empezar-vacunacion-primera-quincena-enero_0_gD2Mt_qmL.html

110. La muerte de Maradona: denunciaron a Alberto Fernández por el velatorio y lo acusaron de favorecer la propagación del coronavirus(Nota periodística, 28/11/20) Disponible en: https://www.lanacion.com.ar/politica/opositores-denunciaron-alberto-fernandez-velatorio-maradona-nid2522891

111. Luis Cámera: “El velatorio multitudinario de Maradona en Plaza de Mayo traerá más contagios de COVID-19" (Nota periodística, 30/11/20) Disponible en: https://www.infobae.com/salud/2020/11/30/luis-camera-el-velatorio-multitudinario-de-maradona-en-plaza-de-mayo-traera-mas-contagios-de-covid-19/

112. Informes de Situación COVID. Municipalidad de Rosario. Disponibles en https://www.rosario.gob.ar/web/coronavirus/coronavirus-informesde-situacion-y-tableros)

113. Los peligros de la sensación "el coronavirus ya fue" (Nota periodística, 15/11/20) Disponible en: https://www.paginal2.com.ar/305995-lospeligros-de-la-sensacion-el-coronavirus-ya-fue?fbclid=IwAR3-96yWlszrl4Qwl4PKKP9Vd7PWJDxHjMJhjl7_2itsBHsx852SVcbDiHg 